\title{
Three-Phase Distribution System Load Flow Analysis Using Sequence Components
}

\author{
Rudy Gianto
}

\begin{abstract}
Electric power distribution systems are usually very unbalance. Therefore, a power flow method that can handle the three-phase configuration of the power system is needed so that the system planning and operation can properly be carried out. In the case of three-phase distribution system power flow analysis, for each system bus (except for substation bus), the voltage magnitude and angle of the three phases must be calculated. These calculations are carried out under certain loading conditions. After these voltages have been calculated, the electric power flows and losses in the distribution lines, and the substation power can also be determined. This paper proposes a new technique for three-phase distribution system power flow analysis using sequence components. The new formulation for the power flow problem in terms of sequence components is also proposed and developed in this paper. The application of sequence components has the advantage that the size of the problem can effectively be reduced, and solution to the power flow problem will be easier to find. Case study using a representative distribution test system confirms the validity of the proposed method where comparative studies between the proposed (i.e. sequence components based) method and the phase components based method are carried out.
\end{abstract}

Index Terms - Electric distribution network, unbalance system, load flow analysis, sequence components.

\section{INTRODUCTION}

Power flow (or load flow) is an important tool in operational study and planning of electric power systems. The single-phase power flow algorithm assumes that the unbalance in an electric power system is small and can be neglected so that the system can be considered to be balanced. There are many cases where system unbalance can no longer be ignored. This can occur for example due to: (i) load unbalance, (ii) transmission/distribution network unbalance, and (iii) combination of (i) and (ii). Thus, a three-phase power flow method that can be used on an unbalanced system is needed so that the system can be analyzed.

Several researchers have developed some interesting three-phase power flow algorithms to analyze the unbalanced distribution systems. These algorithms can be grouped into two categories: (i) algorithms that use the Newton-Raphson iteration scheme [1]-[5]; and (ii) algorithms that utilize the radial structure of the power distribution system (forward/backward sweeping techniques) [6]-[11].

Unbalanced power systems can be modeled using phase

Published on August 4, 2020.

Rudy Gianto, Department of Electrical Engineering, Tanjungpura

University, Indonesia.

(corresponding e-mail: rudy.gianto@ee.untan.ac.id) components. However, the application of sequence components has the advantage that the size of the problem can effectively be reduced. For power systems with $\mathrm{n}$ buses, this size will be reduced from $3 n \times 3 n$ admittance matrix (i.e. phase admittance matrix) to three $\mathrm{n} \times \mathrm{n}$ admittance matrices (i.e. zero, positive and negative sequence admittance matrices). With this reduction, the complexity of the power flow problem formulation will also be reduced, and therefore, it will be easier to solve.

The present work investigates the three-phase distribution system load flow (DSLF) method using sequence components. The new formulation for the power flow problem in terms of sequence components is proposed and developed in this paper. Comparative studies between the proposed method and the phase components based method are also carried out. These comparative studies are conducted to validate the proposed power flow method using sequence components.

\section{THEORY OF SEQUENCE COMPONENTS}

\section{A. Voltage and Current}

By using the symmetrical component theory, phase voltage and current can be expressed in terms of sequence voltage and current as follows [12]:

$$
\begin{aligned}
& \mathbf{V}^{a b c}=\mathbf{A} \mathbf{V}^{012} \\
& \mathbf{I}^{a b c}=\mathbf{A} \mathbf{I}^{012}
\end{aligned}
$$

where:

$$
\begin{aligned}
& \mathbf{V}^{a b c}=\left[\begin{array}{l}
V^{a} \\
V^{b} \\
V^{c}
\end{array}\right] ; \mathbf{V}^{012}=\left[\begin{array}{c}
V^{0} \\
V^{1} \\
V^{2}
\end{array}\right] \\
& \mathbf{I}^{a b c}=\left[\begin{array}{c}
I^{a} \\
I^{b} \\
I^{c}
\end{array}\right] ; \mathbf{I}^{012}=\left[\begin{array}{c}
I^{0} \\
I^{1} \\
I^{2}
\end{array}\right] \\
& \mathbf{A}=\left[\begin{array}{ccc}
1 & 1 \\
1 & 1 \angle 240^{o} & 1 \angle 120^{o} \\
1 & 1 \angle 120^{o} & 1 \angle 240^{o}
\end{array}\right]
\end{aligned}
$$

Based on (1) and (2), it can be shown that, in terms of phase voltage and current, the formulations of sequence 
voltage and current are:

$$
\begin{aligned}
& \mathbf{V}^{012}=\mathbf{A}^{-1} \mathbf{V}^{a b c} \\
& \mathbf{I}^{012}=\mathbf{A}^{-1} \mathbf{I}^{a b c}
\end{aligned}
$$

where:

$$
\mathbf{A}^{-1}=\frac{1}{3}\left[\begin{array}{ccc}
1 & 1 & 1 \\
1 & 1 \angle 120^{\circ} & 1 \angle 240^{\circ} \\
1 & 1 \angle 240^{\circ} & 1 \angle 120^{\circ}
\end{array}\right]
$$

\section{B. Impedance}

The following is the derivation of phase and sequence impedance matrices for a three-phase electrical network. Since:

$$
\mathbf{V}^{a b c}=\mathbf{Z}^{a b c} \mathbf{I}^{a b c}
$$

where:

$$
\mathbf{Z}^{a b c}=\left[\begin{array}{lll}
Z^{a a} & Z^{a b} & Z^{a c} \\
Z^{b a} & Z^{b b} & Z^{b c} \\
Z^{c a} & Z^{c b} & Z^{c c}
\end{array}\right]
$$

Then, by multiplying both sides of (9) with $\mathbf{A}^{-1}$, (9) can be rewritten as:

$$
\mathbf{A}^{-1} \mathbf{V}^{a b c}=\mathbf{A}^{-1} \mathbf{Z}^{a b c} \mathbf{I}^{a b c}
$$

On using (2) and (6) in (11) will result in:

$$
\mathbf{V}^{012}=\mathbf{Z}^{012} \mathbf{I}^{012}
$$

where $\mathbf{Z}^{012}$ is the sequence impedance matrix and has the following formulation:

$$
\mathbf{Z}^{012}=\mathbf{A}^{-1} \mathbf{Z}^{a b c} \mathbf{A}
$$

\section{Power}

For three-phase power system, complex powers ini each phase are calculated using:

$$
\begin{aligned}
& S^{a}=V^{a} I^{a *} \\
& S^{b}=V^{b} I^{b *} \\
& S^{c}=V^{c} I^{c^{*}}
\end{aligned}
$$

Based on (1) and (2), the above complex power equations can be rewritten in terms of sequence voltage and current as:

$$
S^{a}=\left(V^{0}+V^{1}+V^{2}\right)\left(I^{0}+I^{1}+I^{2}\right)^{*}
$$

$$
\begin{aligned}
& S^{b}=\left(V^{0}+a^{2} V^{1}+a V^{2}\right)\left(I^{O}+a^{2} I^{1}+a I^{2}\right)^{*} \\
& S^{c}=\left(V^{0}+a V^{1}+a^{2} V^{2}\right)\left(I^{O}+a I^{1}+a^{2} I^{2}\right)^{*}
\end{aligned}
$$

where: $a=1 \angle 120^{\circ}$ is the complex operator.

In vector/matrix form, $(15)$ is formulated as:

$$
\mathbf{S}^{a b c}=\operatorname{diag}\left(\mathbf{A V}^{012}\right)\left(\mathbf{A I}^{012}\right)^{*}
$$

where: $\mathbf{S}^{a b c}=\left[\begin{array}{lll}S^{a} & S^{b} & S^{c}\end{array}\right]^{T}$.

\section{THREE-PHASE DSLF FORMULATION}

\section{A. Sequence Admittance of Distribution Line}

As explained previously, the unbalance in power system comes from two things: (i) line/network unbalance, and/or (ii) load unbalance. However, for power distribution systems, the load unbalance is more dominant than the line unbalance. Therefore, in this paper, the distribution line or network will be considered to be balanced. This means that the line is assumed to be transposed or has the same phase spacing so that the mutual impedances are identical (or: $Z_{a b}$ $=\mathrm{Z}_{\mathrm{bc}}=\mathrm{Z}_{\mathrm{ac}}=\mathrm{Z}_{\mathrm{m}}$ ). Moreover, the self impedances are also assumed to be the same (or: $\mathrm{Z}_{\mathrm{aa}}=\mathrm{Z}_{\mathrm{bb}}=\mathrm{Z}_{\mathrm{cc}}=\mathrm{Z}_{\mathrm{s}}$ ). Therefore, based on (10), the phase impedance matrix becomes [12]:

$$
\mathbf{Z}^{a b c}=\left[\begin{array}{ccc}
Z^{s} & Z^{m} & Z^{m} \\
Z^{m} & Z^{s} & Z^{m} \\
Z^{m} & Z^{m} & Z^{s}
\end{array}\right]
$$

On using (13), it can be shown that the phase impedance matrix (17) when converted into its sequence impedance matrix will have the form:

$$
\mathbf{Z}^{012}=\left[\begin{array}{ccc}
Z^{0} & 0 & 0 \\
0 & Z^{1} & 0 \\
0 & 0 & Z^{2}
\end{array}\right]
$$

where:

$$
\begin{aligned}
& Z^{0}=Z^{s}+2 Z^{m} \\
& Z^{1}=Z^{2}=Z^{s}-Z^{m}
\end{aligned}
$$

Furthermore, the sequence admittance matrix that corresponds to the sequence impedance matrix (18) is:

$$
\mathbf{Y}^{012}=\left(\mathbf{z}^{012}\right)^{-1}=\left[\begin{array}{ccc}
Y^{0} & 0 & 0 \\
0 & Y^{1} & 0 \\
0 & 0 & Y^{2}
\end{array}\right]
$$

where: 


$$
\begin{aligned}
& Y^{0}=\frac{1}{Z^{s}+2 Z^{m}} \\
& Y^{l}=Y^{2}=\frac{1}{Z^{s}-Z^{m}}
\end{aligned}
$$

\section{B. Load Flow Problem Formulation}

On using (12) and (20), the bus (nodal) voltage/current equation in terms of sequence components can be written as:

$$
\mathbf{I}^{012}=\mathbf{Y}^{012} \mathbf{V}^{012}
$$

Since $\mathbf{Y}^{012}$ is a diagonal matrix, (22) can be decoupled into three separate equations as follows:

$$
\begin{aligned}
& I^{0}=Y^{0} V^{0} \\
& I^{1}=Y^{1} V^{1} \\
& I^{2}=Y^{2} V^{2}
\end{aligned}
$$

Based on (23), for distribution system with multiple buses, the nodal equations in terms of sequence components are:

$$
\begin{aligned}
& \mathbf{I}^{0}=\mathbf{Y}^{0} \mathbf{V}^{0} \\
& \mathbf{I}^{1}=\mathbf{Y}^{1} \mathbf{V}^{1} \\
& \mathbf{I}^{2}=\mathbf{Y}^{2} \mathbf{V}^{2}
\end{aligned}
$$

where:

$\mathbf{Y}^{0}, \mathbf{Y}^{1}, \mathbf{Y}^{2}$ : zero, positive, and negative sequence bus admittance matrices

$\mathbf{V}^{0}, \mathbf{V}^{1}, \mathbf{V}^{2}$ : zero, positive, and negative sequence bus voltage vectors

$\mathbf{I}^{0}, \mathbf{I}^{1}, \mathbf{I}^{2}$ : zero, positive, and negative sequence bus current vectors

For distribution system with $\mathrm{n}$ buses, the above bus admittance matrices, bus voltage vectors, and bus current vectors will have the forms:

$$
\begin{aligned}
\mathbf{Y}^{k} & =\left[\begin{array}{cccc}
Y_{11}^{k} & Y_{12}^{k} & \cdots & Y_{1 n}^{k} \\
Y_{21}^{k} & Y_{22}^{k} & \cdots & Y_{2 n}^{k} \\
\vdots & \vdots & \ddots & \vdots \\
Y_{n 1}^{k} & Y_{n 2}^{k} & \cdots & Y_{n n}^{k}
\end{array}\right] \\
\mathbf{V}^{k} & =\left[\begin{array}{c}
V_{1}^{k} \\
V_{2}^{k} \\
\vdots \\
V_{n}^{k}
\end{array}\right] ; \mathbf{I}^{k}=\left[\begin{array}{c}
I_{1}^{k} \\
I_{2}^{k} \\
\vdots \\
I_{n}^{k}
\end{array}\right]
\end{aligned}
$$

On the other hand, based on (16), the complex power at each bus of electrical distribution system is formulated as:

$$
\begin{aligned}
& \mathbf{S}_{G i}^{a b c}-\mathbf{S}_{L i}^{a b c}=\operatorname{diag}\left(\mathbf{A} \mathbf{V}_{i}^{012}\right)\left(\mathbf{A I} \mathbf{I}_{i}^{012}\right)^{*} \\
& \text { with } i=1,2, \ldots, n
\end{aligned}
$$

where $\mathbf{S}_{\mathrm{Gi}}$ and $\mathbf{S}_{\mathrm{Li}}$ are power generation and load at i-th bus, respectively.

Rearranging (24) and (26) will results in:

$$
\begin{aligned}
& \mathbf{I}^{0}-\mathbf{Y}^{0} \mathbf{V}^{0}=\mathbf{0} \\
& \mathbf{I}^{1}-\mathbf{Y}^{1} \mathbf{V}^{1}=\mathbf{0} \\
& \mathbf{I}^{2}-\mathbf{Y}^{2} \mathbf{V}^{2}=\mathbf{0} \\
& \mathbf{S}_{G i}^{a b c}-\mathbf{S}_{L i}^{a b c}-\operatorname{diag}\left(\mathbf{A} \mathbf{V}_{i}^{012}\right)\left(\mathbf{A I}_{i}^{012}\right)^{*}=\mathbf{0}
\end{aligned}
$$

Set of equations (27) is the formulation of power flow problem for three-phase distribution system in the forms of sequence components. In power flow analysis, the set of equations (27) must be solved simultaneously. The results are then used to evaluate the steady state performance of the distribution system. In summary, all of the quantities in the power flow formulation, i.e. the quantities to be calculated (unknown quantities) and the specified quantities (known quantities), for each system bus are shown in Table I.

TABLE I: KNOWN AND UNKNOWN QUANTITIES

\begin{tabular}{ccc}
\hline \hline Bus & Known Quantities & Unknown Quantities \\
\hline & $\mathbf{Y}^{0} ; \mathbf{Y}^{1} ; \mathbf{Y}^{2} ; \mathbf{S}_{L}^{a b c}$ & $I^{0} ; I^{1} ; I^{2}$ \\
Substation & $\mathbf{V}^{012}=\left[\begin{array}{c}0 \\
1 \angle 0^{o} \\
0\end{array}\right]$ & $\mathbf{S}_{G}^{a b c}$ \\
\hline Load & $\mathbf{Y}^{0} ; \mathbf{Y}^{1} ; \mathbf{Y}^{2} ; \mathbf{S}_{L}^{a b c}$ & $I^{0} ; I^{1} ; I^{2}$ \\
& $\mathbf{S}_{G}^{a b c}=\left[\begin{array}{ccc}0 & 0 & 0\end{array}\right]^{T}$ & $V^{0} ; V^{1} ; V^{2}$
\end{tabular}

\section{Solution Technique and Starting Values}

Since sets of equations (27) are nonlinear, iterative techniques (such as Newton-Raphson method or trust-region method) have to be used to solve the equations [13], [14]. To initialize the iteration process, the following starting values for the unknown variables can be applied.

- Substation power:

$$
\mathbf{S}_{G}^{a b c(0)}=\sum \mathbf{S}_{L i}^{a b c} \quad \text { (total load in the system) }
$$

- Load bus voltages:

where: $\mathrm{k}=0,1,2$. 


$$
\mathbf{V}_{i}^{012(0)}=\left[\begin{array}{c}
0 \\
1 \angle 0^{\circ} \\
0
\end{array}\right]
$$

- Bus currents:

$$
\mathbf{I}_{i}^{012(0)}=\left[\begin{array}{c}
0 \\
\sum_{j=1}^{n} Y_{i j}^{l} \\
0
\end{array}\right]
$$

\section{CAse Study}

\section{A. Test System}

In case study, 10-bus distribution system as shown in Fig. 1 will be used to validate the proposed method. This system is adopted from [6] and has a system voltage of $8.66 \mathrm{kV}$. Data for this test system are shown in Tables II and III. It should also be noted that all of the calculations are performed on a personal computer (PC) with the help of MATLAB $^{\mathrm{TM}}$ software.

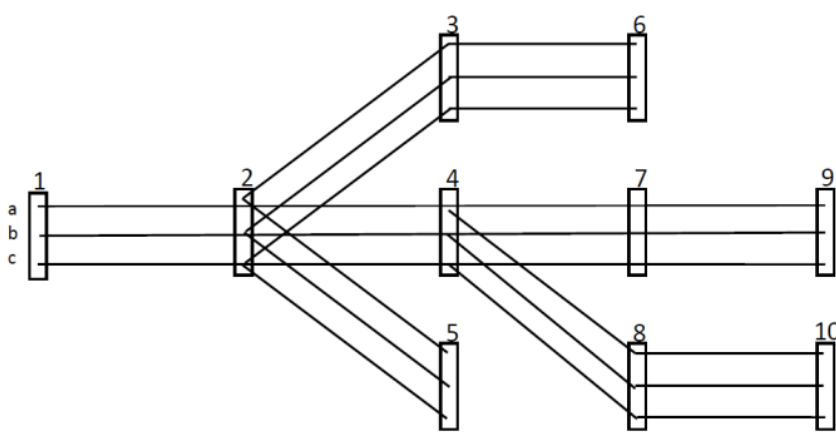

Fig. 1. 10-bus distribution test system.

TABLE II: 10-BUS SYSTEM LINE IMPEDANCE (OHM)

\begin{tabular}{cccc}
\hline \hline No. & Line & Self Impedance & Mutual Impedance \\
\hline 1 & $1-2$ & $1+\mathrm{j} 2$ & $\mathrm{j} 0.5$ \\
2 & $2-3$ & $1+\mathrm{j}$ & $\mathrm{j} 0.25$ \\
3 & $2-4$ & $1+\mathrm{j} 2$ & $\mathrm{j} 0.5$ \\
4 & $2-5$ & $1+\mathrm{j} 2$ & $\mathrm{j} 0.5$ \\
5 & $3-6$ & $1+\mathrm{j} 2$ & $\mathrm{j} 0.5$ \\
6 & $4-7$ & $1+\mathrm{j}$ & $\mathrm{j} 0.25$ \\
7 & $4-8$ & $1+\mathrm{j}$ & $\mathrm{j} 0.25$ \\
8 & $7-9$ & $1+\mathrm{j}$ & $\mathrm{j} 0.25$ \\
9 & $8-10$ & $1+\mathrm{j} 2$ & $\mathrm{j} 0.5$ \\
\hline \hline
\end{tabular}

\begin{tabular}{ccccccc}
\multicolumn{8}{c}{ TABLE III: 10-BUS SYSTEM LOAD (KW, KVAR) } \\
\hline \hline \multirow{2}{*}{ Bus } & \multicolumn{2}{c}{ Phase a } & \multicolumn{2}{c}{ Phase b } & \multicolumn{2}{c}{ Phase c } \\
\cline { 2 - 7 } & $\mathrm{P}$ & $\mathrm{Q}$ & $\mathrm{P}$ & $\mathrm{Q}$ & $\mathrm{P}$ & $\mathrm{Q}$ \\
\hline 1 & 0 & 0 & 0 & 0 & 0 & 0 \\
2 & 50 & 25 & 50 & 25 & 50 & 12.5 \\
3 & 50 & 25 & 37.5 & 12.5 & 37.5 & 25 \\
4 & 50 & 25 & 25 & 25 & 25 & 25 \\
5 & 37.5 & 25 & 37.5 & 12.5 & 50 & 12.5 \\
6 & 100 & 75 & 50 & 75 & 100 & 50 \\
7 & 100 & 75 & 100 & 12.5 & 25 & 12.5 \\
8 & 100 & 75 & 25 & 12.5 & 50 & 25 \\
9 & 10 & 12.5 & 10 & 25 & 25 & 12.5 \\
10 & 10 & 25 & 25 & 12.5 & 10 & 12.5 \\
\hline \hline
\end{tabular}

\section{B. Results and Discussion}

Results of the power flow study in terms of bus voltages are shown in Table IV. For comparison purposes, results from the phase components based power flow method are also shown in Table V. It can be seen that the results are in exact agreement which confirms the validity of the proposed method.

TABel IV: VolTAge PROFILE OF 10-Bus Distribution NeTWORK (PROPOSED METHOD)

\begin{tabular}{cccc}
\hline \hline Bus & $\mathrm{V}_{\mathrm{a}}$ & $\mathrm{Vb}_{\mathrm{b}}$ & $\mathrm{V}_{\mathrm{c}}$ \\
\hline 1 & $1.00000 \angle 0.00^{0}$ & $1.00000 \angle-120.00^{0}$ & $1.00000 \angle 120.00^{0}$ \\
2 & $0.95072 \angle-1.13^{0}$ & $0.97586 \angle-120.86^{0}$ & $0.97205 \angle 119.37^{0}$ \\
3 & $0.94132 \angle-1.17^{0}$ & $0.96967 \angle-120.79^{0}$ & $0.96357 \angle 119.30^{0}$ \\
4 & $0.92114 \angle-1.79^{0}$ & $0.96670 \angle-120.44^{0}$ & $0.96070 \angle 119.36^{0}$ \\
5 & $0.94750 \angle-1.20^{0}$ & $0.97347 \angle-120.97^{0}$ & $0.96934 \angle 119.22^{0}$ \\
6 & $0.93278 \angle-1.38^{0}$ & $0.96274 \angle-120.76^{0}$ & $0.95584 \angle 119.03^{0}$ \\
7 & $0.91226 \angle-1.79^{0}$ & $0.96175 \angle-120.61^{0}$ & $0.95822 \angle 119.39^{0}$ \\
8 & $0.91234 \angle-1.78^{0}$ & $0.96482 \angle-120.49^{0}$ & $0.95674 \angle 119.39^{0}$ \\
9 & $0.91160 \angle-1.76^{0}$ & $0.96029 \angle-120.56^{0}$ & $0.95685 \angle 119.36^{0}$ \\
10 & $0.90970 \angle-1.75^{0}$ & $0.96315 \angle-120.58^{0}$ & $0.95595 \angle 119.41^{0}$ \\
\hline \hline
\end{tabular}

TABel V: Voltage Profile of 10-Bus Distribution NeTwork (PHASE COMPONENTS METHOD)

\begin{tabular}{cccc}
\hline \hline Bus & $\mathrm{V}_{\mathrm{a}}$ & $\mathrm{V}_{\mathrm{b}}$ & $\mathrm{V}_{\mathrm{c}}$ \\
\hline 1 & $1.00000 \angle 0.00^{0}$ & $1.00000 \angle-120.00^{0}$ & $1.00000 \angle 120.00^{0}$ \\
2 & $0.95072 \angle-1.13^{0}$ & $0.97586 \angle-120.86^{0}$ & $0.97205 \angle 119.37^{0}$ \\
3 & $0.94132 \angle-1.17^{0}$ & $0.96967 \angle-120.79^{0}$ & $0.96357 \angle 119.30^{0}$ \\
4 & $0.92114 \angle-1.79^{0}$ & $0.96670 \angle-120.44^{0}$ & $0.96070 \angle 119.36^{0}$ \\
5 & $0.94750 \angle-1.20^{0}$ & $0.97347 \angle-120.97^{0}$ & $0.96934 \angle 119.22^{0}$ \\
6 & $0.93278 \angle-1.38^{0}$ & $0.96274 \angle-120.76^{0}$ & $0.95584 \angle 119.03^{0}$ \\
7 & $0.91226 \angle-1.79^{0}$ & $0.96175 \angle-120.61^{0}$ & $0.95822 \angle 119.39^{0}$ \\
8 & $0.91234 \angle-1.78^{0}$ & $0.96482 \angle-120.49^{0}$ & $0.95674 \angle 119.39^{0}$ \\
9 & $0.91160 \angle-1.76^{0}$ & $0.96029 \angle-120.56^{0}$ & $0.95685 \angle 119.36^{0}$ \\
10 & $0.90970 \angle-1.75^{0}$ & $0.96315 \angle-120.58^{0}$ & $0.95595 \angle 119.41^{0}$ \\
\hline \hline
\end{tabular}

\section{CONCLUSION}

Power flow is an important tool in operational study and planning of electric power systems. The single-phase power flow algorithm assumes that the unbalance in an electric power system is small and can be neglected so that the system can be considered to be balanced. There are many cases where system unbalance can no longer be ignored, and therefore, three-phase power flow algorithm has to be used in the analysis. A new technique for three-phase distribution system power flow analysis using sequence components has been proposed and developed in this paper. The application of sequence components has the advantage that the size of the problem can effectively be reduced, and solution to the power flow problem will be easier to find. Comparative studies between the proposed method and the phase components based method have also been carried out to validate the proposed power flow method.

\section{REFERENCES}

[1] P.A.N. Garcia, et al., "Three-Phase Power Flow Calculations Using the Currents Injection Methods", IEEE Trans. Power Systems, vol. 15, no. 2, pp. 508-514, May 2000.

[2] J.H. Teng, and C.Y. Chang, "A Novel and Fast Three-Phase Load Flow for Unbalanced Radial Distribution Systems", IEEE Trans. Power Systems, vol. 17, no. 4, pp. 1238-1244, Nov. 2002.

[3] J.M.T. Marinho, and G.N. Taranto, "A Hybrid Three-Phase SinglePhase Power Flow Formulation", IEEE Trans. Power Systems, vol 23, no. 3, pp. 1063-1070, August 2008. 
[4] N.C. Yang, and H.C. Chen, "Three-Phase Power Flow Solutions Using Decomposed Quasi-Newton Method for Unbalanced Radial Distribution Networks", IET Gen. Trans. \& Dist., vol. 11, no. 4, pp. 3594-3600, Sept. 2017.

[5] B. Sereeter, K. Vuik, and C. Witteveen, "Newton Power Flow Methods for Unbalanced Three-Phase Distribution Networks", Energies, vol. 10, no. 1658, pp. 1-20, 2017.

[6] E.R. Ramos, et al., "Quasi-Coupled Three-Phase Radial Load Flow", IEEE Trans. Power Systems, vol. 19, no. 2, pp. 776-781, May 2004.

[7] G.W. Chang, et al., "An Improved Backward/Forward Sweep Load Flow Algorithm for Radial Distribution Systems", IEEE Trans. Power Systems, vol. 22, no. 2, pp. 882-884, May 2007.

[8] J.B.V. Subrahmanyam, et al., "A Simple and Direct Approach for Unbalanced Radial Distribution System Three-Phase Load Flow Solution", Research Journal of Applied Sciences, Engineering and Technology, vol. 2, no. 5, pp. 452-459, May 2010.

[9] U.R. Puthireddy, et al., "Power Flow Analysis of Three Phase Unbalanced Distribution System", International Journal of Advances in Engineering and Technology, vol.3, no.1, pp.514-524, March 2012.

[10] K.N. Maya, and E.A. Jasmin, "A Three-Phase Power Flow Algorithm for Distribution Network Incorporating the Impact of Distributed Generation Models", Procedia Technology, vol. 21, pp. 326-331, 2015.

[11] C. Gonzalez-Moran, and B. Mohamed, "Matrix Backward Forward Sweep for Unbalanced Power Flow in ab0 Frame", Electric Power System Research, Vol. 148, pp. 273-281, Juli 2017.

[12] T. Gonen. Modern Power System Analysis, John Wiley \& Sons Inc., 1988.

[13] R. Gianto, and K.H. Khwee, "A New Method for Load Flow Solution of Electric Power Distribution System," International Review of Electrical Engineering, vol. 11, no. 5, pp. 535-541, 2016.

[14] R. Gianto, "T-Circuit Model of Asynchronous Wind Turbine for Distribution System Load Flow Analysis," International Energy Journal, vol. 19, no. 2, pp. 77-88, 2019.

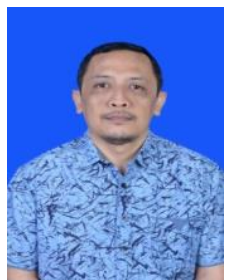

Rudy Gianto was born in Bandung, Indonesia. He received $\mathrm{BE}, \mathrm{ME}$ and $\mathrm{PhD}$ degrees from Tanjungpura University in 1991, Bandung Institute of Technology in 1995, and The University of Western Australia in 2009 respectively. Currently, he is an Associate Professor at Tanjungpura University, Indonesia. His research interests include power system analysis, simulation of power system dynamics, and distributed generation. He has published many research papers in international journals and conferences. 